\title{
Spectral Effects of Pulsations in Blue Supergiants
}

\author{
S. Tomić, M. Kraus and M. E. Oksala \\ Astronomický Ústav AVČR, Fričova 298, 25165 Ondřejov, Czech Republic \\ email: sanja@sunstel.asu.cas.cz
}

\begin{abstract}
We have been spectroscopically monitoring a number of blue supergiants, focusing on several strategic photospheric and wind lines. Our aim is to detect line profile variability, and to determine its origin. Here, we present preliminary results for $\rho$ Leo and $\epsilon$ Ori. We conduct an asteroseismic analysis of HeI $\lambda 6678$. We find in each star multiple periods raging from hours to several days. In addition, we observe strong, night to night variability in $\mathrm{H} \alpha$.
\end{abstract}

Keywords. line: profiles, stars: oscillations, supergiants, stars: early-type

\section{Introduction}

Blue supergiants (BSGs) are luminous, evolved massive stars. Their photospheric lines are broadened by both stellar rotation and so-called macroturbulence. The nature of this often large macroturbulence (Simón-Díaz \& Herrero 2014) is not clear yet, but has been suggested to be connected to stellar pulsations (Aerts et al. 2009). So far, only a few BSGs have been reported to pulsate (Lefever et al. 2007; Saio et al. 2006; Kraus et al. 2012). However, BSGs are well known to display strong variability of their wind lines such as $\mathrm{H} \alpha$, which might indicate a connection between pulsations and variable wind conditions.

We initiated an observing campaign to monitor spectroscopically a sample of northern Galactic BSGs. Our goal is to identify pulsation periods and their possible link to variable mass-loss. For this study we focus on two objects, $\rho$ Leo and $\epsilon$ Ori. Both are known to show variability of their optical line profiles.

\section{Observations, data analysis and preliminary results}

With the Perek $2 \mathrm{~m}$ telescope at the Ondřejov Observatory, we obtained 394 spectra of $\epsilon$ Ori and 254 spectra of $\rho$ Leo with SNR $>300$ in the period 2014 February 6 to March 14. Spectral time series were obtained for both stars during the final seven nights of the observing run. The observations include $\mathrm{H} \alpha$ and HeI $\lambda 6678$.

Clearly, both lines display strong profile variability. $\mathrm{H} \alpha$ changes from night to night in both shape and strength (Tomić et al., in preparation). We focus on the HeI $\lambda 6678$ line and compute the first three moments of its profile. The first and third moment vary in phase (Fig. 1) indicating pulsations. We apply the Lomb-Scargle method for the period analysis. For both stars, the identified frequencies, their amplitudes, and their phases are listed in Table 1. For $\rho$ Leo, we identified 9 periods ranging from $\sim 5$ hours to $\sim 6$ days. For $\epsilon$ Ori, we find 13 periods ranging from $\sim 2$ hours to $\sim 3$ days. We prewhitened the data with each identified period and made a final fitting using a least-square method.

\section{Acknowledgements}

This work was supported by GAČR (14-21373S), MŠMT (LG14013) and RVO:67985815. 

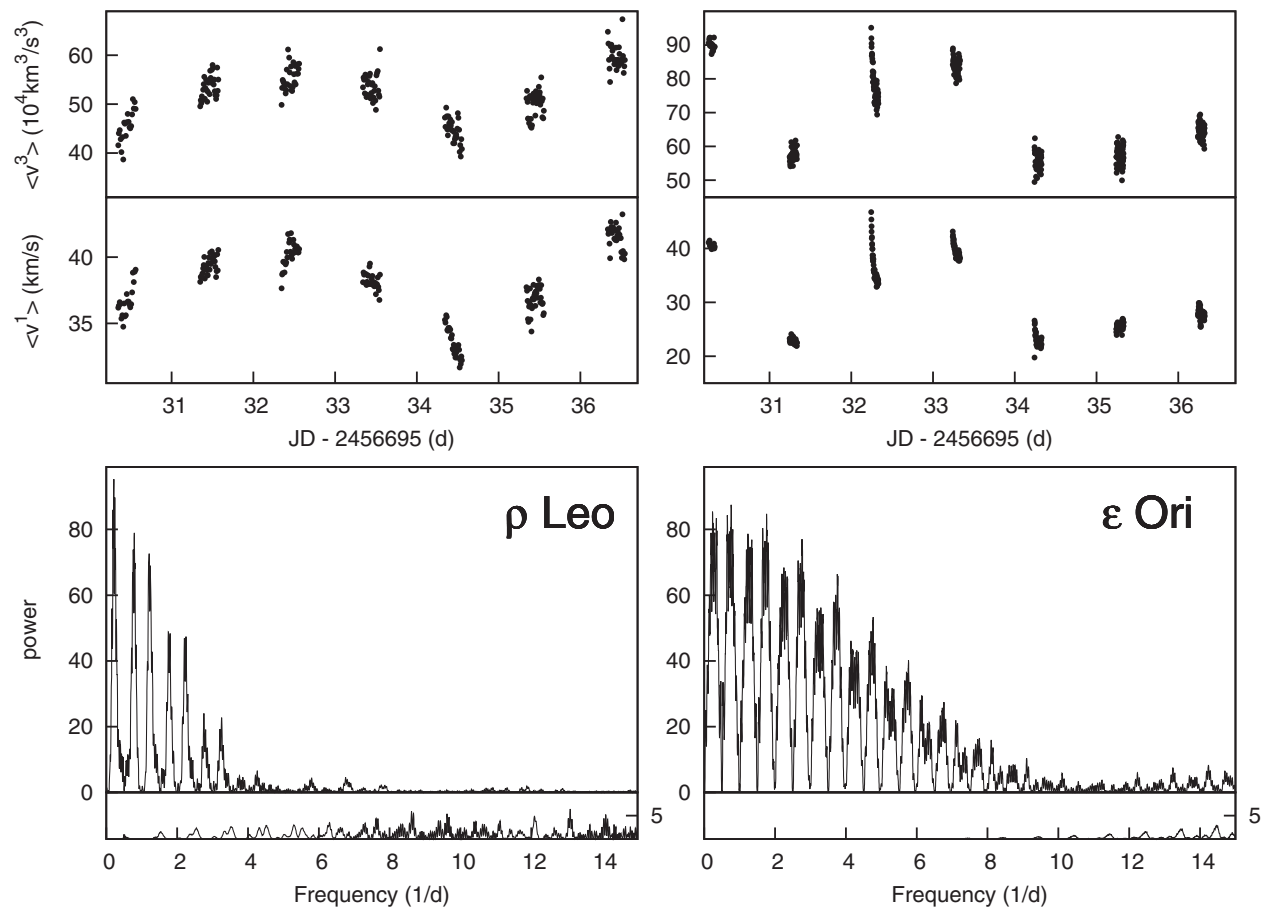

Figure 1. Top: Variability in the first and third moment during seven consecutive nights. Bottom: Lomb-Scargle periodograms of the first moments and their residuals.

Table 1. Lists of identified frequencies

\begin{tabular}{ccc|ccc}
\hline \multicolumn{2}{c|}{$\rho$ Leo } & \multicolumn{3}{c}{$\epsilon$ Ori } \\
\hline $\begin{array}{c}\text { Frequency } \\
1 / \mathrm{d}\end{array}$ & $\begin{array}{c}\text { amplitude } \\
\mathrm{km} / \mathrm{s}\end{array}$ & phase & $\begin{array}{c}\text { Frequency } \\
1 / \mathrm{d}\end{array}$ & $\begin{array}{c}\text { amplitude } \\
\mathrm{km} / \mathrm{s}\end{array}$ \\
\hline 0.79 & 6.43 & 4.26 & 3.75 & -2.89 & 3.45 \\
1.25 & 2.45 & 4.77 & 2.81 & -8.09 & 5.36 \\
0.16 & 5.98 & 1.48 & 1.35 & -9.13 & 7.08 \\
2.22 & -3.02 & -5.06 & 4.70 & 1.97 & -0.53 \\
2.78 & 1.47 & -1.02 & 1.64 & 22.97 & 2.75 \\
0.37 & 0.91 & 0.80 & 0.44 & 4.77 & 9.12 \\
0.89 & 5.77 & 5.27 & 0.62 & -30.28 & 1.67 \\
1.87 & 2.79 & 1.83 & 2.20 & 11.35 & 11.35 \\
4.73 & 0.44 & 4.73 & 4.19 & 6.99 & -4.41 \\
& & & 3.09 & 6.10 & 3.25 \\
& & & 5.77 & 2.97 & 1.15 \\
& & & 5.13 & 1.57 & 6.05 \\
& & & 10.99 & -1.07 & 3.47
\end{tabular}

\section{References}

Aerts, C., Puls, J., Godart, M., \& Dupret, M.-A. 2009, A\&A 508, 409

Kraus, M., Tomić, S., Oksala, M. E., \& Smole, M. 2012, A\&3A 542, L32

Lefever, K., Puls, J., \& Aerts, C. 2007, A\&A 463, 1093

Saio, H., Kuschnig, R., Gautschy, A., et al. 2006, ApJ 650, 1111

Simón-Díaz, S. \& Herrero, A. 2014, A\&\&A 562, A135 